\title{
Geometry of a Relativistic Quantum Chaos: New approach to dynamics of quantum systems in elec- tromagnetic field and some applications
}

\author{
A.V. Glushkov, V.B. Ternovsky, G.P. Prepelitsa
}

\begin{abstract}
Work goes on development of new relativistic chaos-geometric and quantum-dynamic approach to solve problems of complete modelling relativistic chaotic dynamics of atoms in an electromagnetic field. As usually an approach consistently includes a number of new relativistic quantum models and a number of new or improved methods of analysis (correlation integral, fractal analysis, algorithms, average mutual information, false nearest neighbors, Lyapunov exponents, surrogate data, non-linear prediction, spectral methods, etc.) We present here firstly the corresponding atomic ionization quantitative data for some atoms in a microwave external field
\end{abstract}

Keywords relativistic quantum chaos, quantum systems in field, chaosgeometric and quantum-dynamics approach

Mathematics Subject Classification (2000) 55R01-55B13

UDK 517.9

\section{Introduction}

This paper goes on our investigations of the energy, spectral and geometric features of relativistic quantum systems in an external electromagnetic field and revealed quantum chaos features of the studied systems. The main attention is devoted to quantitative studying the atomic dynamics eigen energy values and quantum chaos fundamental characteristics. The different algorythms of a whole relativcistic energy approach to atoms in an electromagnetic field and chaos- 
geometric approach to studying quantum chaos in the comlex quantum systems [1]-[14].

Let us re remind that dynamics of the cited systems in external electromagnetic field has features of the random, stochastic kind and its realization does not require the specific conditions. The importance of mathematical studying a phenomenon of stochasticity or quantum chaos in dynamical systems is provided by a whole number of technical applications, including a necessity of understanding chaotic features in a work of different electronic devices and systems. New field of investigations of the quantum and other systems has been provided by a great progress in a development of a chaos theory methods. In previous our papers [2-4] we have given a review of new methods and algorithms to analysis of different dynamical systems.

In this paper following [4] we shortly present the theoretical foundations of the new universal relativistic chaos-geometric and quantum-dynamic approach to modelling chaotic dynamics of heavy complex relativistic quantum systems in an external electromagnetic field, opening a new field of relativistic quantum chaos in geometry of a chaos. Chaos-geometric block includes a set of new or partially improved non-linear analysis methods (such as correlation (dimension D) integral, fractal analysis, average mutual information, false nearest neighbours, Lyapunov exponents (LE) and Kolmogorov entropy (KE), power spectrum analysis, the surrogate data, nonlinear prediction, predicted trajectories, neural network methods etc), quantum-dynamical block - new relativistic approach to systems in a field. For a number of heavy systems there are firstly theoretically presented corresponding quantitative ionization dynamics and chaos characteristics.

\section{Relativistic quantum chaos in atomic dynamics in a DC electric and electromagnetic fields}

Our chaos-geometric approach to treating a chaos dynamics has been in details presented earlier [1-3]. Further we present a new relativistic quantum approach to modeling the chaotic dynamics of atomic systems in a dc electric and ac electromagnetic fields, based on the theory of quasi-stationary quasienergy states, optimized operator perturbation theory, method of model-potential, a complex rotation coordinates algorithm method. The universal chaos-geometric block will be used further to treat the chaotic ionization characteristics for a number of heavy atomic systems.

Let us remind that in the case of the electromagnetic field atomic Hamiltonian is usually as follows: 


$$
H=\frac{1}{2} p^{2}+V_{a t}(r)+z F_{0} \cos (\omega t)
$$

The field is periodic, of course one should use the Floquet theorem; then the eigen Floquet states $\Psi_{E_{j}}(r, t)>$ and quasienergies $E_{j}$ are defined as the eigen functions and eigen values of the Floquet Hamiltonian $H_{F}=H-i \partial_{t}$. In the general form with using the method of complex coordinates the problem reduces to the solution of stationary SchrC†dinger equation, which is as follows in the model potential approximation:

$$
\left(-1 / 2 \cdot \nabla^{2}+V_{a t}(r)+\omega L_{z}+F_{o} z\right) \Psi_{E}(r)=E \Psi_{E}(r)
$$

i.e. to the stationary eigen value and eigen vectors task for some matrix $\mathrm{A}$ (with the consideration of several Floquet zones): $\left(A--E_{j} B\right) E_{j}>=0$. As a decomposition basis, system of the Sturm functions of the operator perturbation theory basis is used.

In our new theory we start from the Dirac Hamiltonian (in relativistic units):

$$
H=\alpha p+\beta-\alpha Z / r_{i}+\sqrt{\alpha} F z
$$

Here a field strength intensity is expressed in the relativistic units $\left(F_{\text {rel }}=\right.$ $\alpha^{5 / 2} F_{\text {at.un. }} ; \alpha$ is the fine structure constant). One could see that a relativistic wave function in the Hilbert space is a bi-spinor. Using the formal transformation of co-ordinates $r \rightarrow r \exp (i \theta)$ in the Hamiltonian

(??), one could get:

$$
H(\theta)=(\alpha c p-Z / r) \exp (-i \theta)+\beta-\sqrt{\alpha} F z \exp (i \theta)
$$

In comparison with an analogous non-relativistic theory, here there is arisen a technical problem. In formulae (3) there is term $\beta$, which can not be simply transformed. One of the solving receptions os a limitation of a sub-space of the Hamiltonian eigen-functions by states of the definite symmetry (momentum $J$ and parity $\mathrm{P}$ ). Thus states can be described by the following functions:

$$
\Psi_{P J}^{M}=1 / r\left(\begin{array}{l}
f(r) \Upsilon_{l J}^{M}(n, \sigma) \\
g(r) \Upsilon_{l^{\prime} J}^{M}(n, \sigma)
\end{array}\right)
$$

Here $l\left(l^{\prime}\right)$ and spin P... in the coupling scheme give a state with the total momentum $J$ and its projection $M_{J}=M$. Action of the Hamiltonian (3) on the functions (5) with definite $J$ results in: 


$$
\begin{aligned}
& \widehat{H}(\theta) \Psi_{P J}^{M}=\alpha_{r}\left(\widehat{p}_{r}-\frac{i \omega(J+1 / 2)}{r} \beta\right) \exp (-i \theta) \Psi_{P J}^{M}+ \\
& +\left(\beta-\frac{\alpha Z}{r} \exp (-i \theta)-\sqrt{\alpha} F z \exp (-i \theta)\right) \Psi_{P J}^{M}
\end{aligned}
$$

where $\alpha_{r}=\left(\begin{array}{c}0 \ldots . \sigma n \\ \sigma n \ldots 0\end{array}\right), \beta=\left(\begin{array}{c}1 \ldots .0 \\ 0 . .-1\end{array}\right), p_{r}=-i(1 / r)(d / d r) r, \mathbf{n}=\mathbf{r} / r, \sigma$ the Pauli matrices; parameter $\omega=-1$, if $l=J-1 / 2$ and $\omega=1$, if $l=J+1 / 2$.

To further diahonalizuvaty Hamiltonian selecting the correct basis functions in subspace, including selecting the following functions (sleterovskoho vodnyepodibnoho or type):

$$
\begin{aligned}
& \Psi_{P J}^{a, M}=1 / r\left(\begin{array}{c}
F(r) \Upsilon_{l J}^{M}(n, \sigma) \\
0
\end{array}\right) \\
& \Psi_{P J}^{b, M}=1 / r\left(\begin{array}{c}
0 \\
i G(r) \Upsilon_{l^{\prime} J}^{M}(n, \sigma)
\end{array}\right)
\end{aligned}
$$

It is easy to see that the matrix elements (6) will be no-zeroth only between the states with the same $M_{J}$. In fact this moment is a single limitation of the whole approach.

Transformation of co-ordinates in the Pauli Hamiltonian (in comparison with the Schrodinger equation Hamiltonian it contents additional potential term of a magnetic dipole in an external field) can be performed by the analogous way. However, procedure in this case is significantly simplified. The other details of the presented approach can be found in [2-5].

\section{Numerical application and conclussions}

As illustration, below we present some results of our numerical modelling ionization dynamics for Rydberg atoms Rb, Cs, Fr (Rb: $n=50-80 ; C s, F r: n=$ $60-80)$ in a microwave field $\left(F=(1.2-3.2) \cdot 10^{-9} a . u . ; \omega / 2 \pi=8.87,36 H G z\right)$. In particular, in Table 1 (from Ref. [5]) there are presented our new relativistic theory data on dependence of the $\mathrm{Rb}$ ionization probability $\mathrm{P}$ upon the $\mathrm{F}$, interaction time "atom-field". For comparison there are listed the non-relativistic theoretical data by Krug-Buchleitner [15]; Th2 - Glushkov,Prepelitsa etal [3].

Table 1. Dependence of ionization probability $\mathrm{P}$ for $\mathrm{Rb}\left(l_{0}=0, m_{0}=\right.$ $0, n_{0}=60-66$ ) on $n_{0}, F$ (at.units), (other parameters: $t=327.2 \pi / \omega$; frequency $\left.\omega_{c}=\omega / 2 \pi=36 \mathrm{GHz}, 8.87 \mathrm{GHz}\right)$ : Th1- numerical simulation of the SchrC $\nmid$ dinger equation by Krug-Buchleitner [15]; Th2 - Glushkov,Prepelitsa etal [3]; Th3 - this work 


\begin{tabular}{|c|c|c|c|c|c|c|}
\hline & Th.1 & Th.2 & Th.3 & Th.1 & Th.2 & Th.2 \\
\hline$n_{0}$ & $\begin{array}{l}F= \\
3.1 \\
10^{-9} \\
\omega_{c}=36\end{array}$ & $\begin{array}{l}F= \\
3.1 \\
10^{-9} \\
\omega_{c}=36\end{array}$ & $\begin{array}{l}F= \\
3.1 \\
10^{-9} \\
\omega_{c}=36\end{array}$ & $\begin{array}{l}F \\
2.8 \\
10^{-9} \\
\omega_{c} \\
8.87\end{array}$ & $\begin{array}{l}F=3.1 . \\
10^{-9} \\
\omega_{c}=8.87\end{array}$ & $\begin{array}{l}F=3.1 . \\
10^{-9} \\
\omega_{c}=8.87\end{array}$ \\
\hline 60 & 0,25 & 0,252 & 0,27 & 0,20 & 0,21 & 0,24 \\
\hline 63 & 0,36 & 0,358 & 0,38 & 0,30 & 0,31 & 0,33 \\
\hline $64^{*}$ & - & - & 0,36 & - & - & 0,31 \\
\hline 65 & 0,34 & 0,347 & 0,37 & 0,28 & 0,29 & 0,32 \\
\hline 66 & 0,36 & 0,371 & 0,39 & 0,34 & 0,35 & 0,38 \\
\hline
\end{tabular}

Comparison of theoretical results with experimental data by Munich group for Rb: $\left(n_{0}>60, F=(1.2-3.2) \cdot 10^{-9}\right.$ a.u., $\left.\omega / 2 \pi=8.87 G H z\right)[15,16]$ shows that all listed data are in a reasonable agreement with experiment, however, the best accuracy is provided by relativistic theory. In table 2 we firstly present new data on dependence of the $\mathrm{Cs}$ ionization probability $\mathrm{P}$ upon the $\mathrm{F}$, interaction time "atom-field" (the same parameters as in table 1). Unfortunately, here there are no any alternative theoretical or experimental results.

Table 2. Dependence of ionization probability $\mathrm{P}$ for $\mathrm{Cs}\left(l_{0}=0, m_{0}=0, n_{0}=\right.$ $65-75)$ on $n_{0}, F$ (the same parameters as in table 1$)$ : this work

\begin{tabular}{|l|l|l|l|l|}
\hline & this work & this work & this work & this work \\
\hline$n_{0}$ & $F=2.8 \cdot$ & $F=3.1 \cdot$ & $F=2.8 \cdot$ & $F=3.1 \cdot 10^{-9}$ \\
& $10^{-9}$ & $10^{-9}$ & $10^{-9}$ & $8.87 G H z$ \\
& $\omega / 2 \pi=36$ & $\omega / 2 \pi=36$ & $8.87 G H z$ & \\
\hline 65 & 0,37 & 0,40 & 0,33 & 0,37 \\
\hline 68 & 0,46 & 0,49 & 0,43 & 0,47 \\
\hline $71^{*}$ & 0,44 & 0,48 & 0,41 & 0,43 \\
\hline 74 & 0,55 & 0,57 & 0,52 & 0,54 \\
\hline
\end{tabular}

As it has been noted in [5], in whole, our modeling relativistic dynamics of ionization alkali Rydberg states in the microwave field for main quantum numbers $n_{0}\left(n_{0} \sim n^{*}\right)$ there are the local violations of probability smooth growth associated with the complex Floquet spectrum, link between the quasi-stationary states and a continuum, the growing influence of multiphoton resonances. The picture becomes by more complicated due to the single-photon near-resonance transitions with quasi-random detuning from resonance and quantum phase shift due to scattering Rydberg electron on the atomic core. It is in agreement with alternative comments in $[3,15]$. We have also used the chaos-geometric approach 
[5] to estimate parameters of relativistic chaotic dynamics for the Rydberg atoms $\mathrm{Rb}, \mathrm{Cs}$, Fr in microwave field: correlation dimension, LE, KE. In Table 3 [5] there were listed the numerical LE values, Kolmogorov entropy $K_{\text {entr }}$, for three atomic systems Positivity first two LE showers certainly evidence of chaotic dynamics for studied systems in a microwave field. We have constructed the quantitative diagram of effects of the quantum fluctuations, stabilization, destabilization, delocalization and performance of the Kolmogorov-Arnold-Mozer theorem in relativistic atomic dynamics. We have found that the regime of the chaotic ionization for the $\mathrm{Li}, \mathrm{Rb}$ in a microwave field at $\omega_{o}=\omega n_{o}^{3}>0.29(R b), 0.25(C s), 0.16(F r)$ switches to dynamic stabilization one. In whole using relativistic chaos-geometric and quantum-dynamical approaches allow to get the results, which confirm an universality and charm of relativistic chaotic phenomena as in relativistic atomic systems in electromagnetic microwave field as other quantum systems and devices.

\section{References}

1. Gutzwiller M.,Chaos in Classical and Quantum Mechanics.-N.-Y.:Springer-Verlag, 1990.$720 \mathrm{p}$.

2. Glushkov A.V., Svinarenko A.A., Buyadzhi V.V., Zaichko P.A., Ternovsky V.B., Chaosgeometric attractor and quantum neural networks approach to simulation chaotic evolutionary dynamics during perception process// Advances in Neural Networks, Fuzzy Systems and Artificial Intelligence, Series: Recent Advances in Computer Engineering (Gdansk,EU).-2014.-Vol.21.-P.143-150.

3. Glushkov A.V., Khetselius O.Yu., Svinarenko A.A., Prepelitsa G.P.Energy approach to atoms in a laser field and quantum dynamics with laser pulses of different shape///In: Coherence and Ultrashort Pulsed Emission, Ed. Duarte F. J. (Intech, Vienna).-2011.P.159-186.

4. Glushkov A.V., Prepelitsa G.P., Lepikh Ya.I., Buyadzhi V.V., Ternovsky V.B., Zaichko P.A., Chaotic dynamics of non-linear processes in atomic and molecular systems in electromagnetic field and semiconductor and fiber laser devices: new approaches, uniformity and charm of chaos// Sensor Electronics and Microsystems Techn.-2014.-Vol.11,N4.-P.43-57.

5. Glushkov A.V., Ternovsky V.B., Buyadzhi V.V., Prepelitsa G.P., Geometry of a Relativistic Quantum Chaos: New approach to dynamics of quantum systems in electromagnetic field and uniformity and charm of a chaos// International Scientific Journal "Proceedings of International Geometry Center".- 2014.-Vol.7,N4.-P.60-71.

6. Gallager R.G., Information theory and reliable communication, Wiley, New York.-1986.

7. Kennel M., Brown R., Abarbanel H., Determining embedding dimension for phase-space reconstruction using geometrical construction//Phys.Rev.A.-1992.-Vol.45.-P.3403-3411.

8. Packard N., Crutchfield J., Farmer J., Shaw R., Geometry from time series// Phys.Rev.Lett-1988.-Vol.45.-P.712-716.

9. Grassberger P., Procaccia I., Measuring the strangeness of strange attractors// Physica D.-1983.-Vol.9.-P.189-208.

10. Fraser A., Swinney H., Independent coordinates for strange attractors from mutual information// Phys Rev A.-1986.-Vol.33.-P.1134-1140.

11. Takens F., Detecting strange attractors in turbulence. In: Dynamical systems and turbulence, ed. Rand D., Young L.- (Springer, Berlin-New York).-1981.-P.366-381.

12. Zimmerman M., Littman M., Kash M., Kleppner D., Stark and Zeemane structure of Rydberg states of alkali-metal atoms// Phys.Rev.A.-1979.-V.20,N6.-P.2251-2275.

13. Gasati G., Guarneri I., Mantica G., Classical Stabilization of periodically kicked hydrogen atoms // Phys.Rev.A.-1994.-V.50(??).-P.5018-5024. 
14. Glushkov A.V., Ivanov L.N., DC Strong-Field Stark-Effect: consistent quantummechanical approach // J.Phys.B:At.Mol.Opt.Phys.-1993.-V.26.-P.L379-388.

15. Krug A., Buchleitner A., Microwave ionization alkali-metal Rydberg states in a realistic numerical experiment//Phys. Rev. A.-2002.-Vol.66.-P.053416 (10p.);

16. Gallagher T., Mahon C., Dexter J., Pillet P., Ionization of sodium and lithium Rydberg atoms by 10-MHz to 15-GHz electric fields//Phys. Rev. A.-1991.- Vol.44.-P. 1859-1872.

\author{
A.V. Glushkov, V.B. Ternovsky, G.P. Prepelitsa \\ Odessa State Environmental University, 15, L' vovskaya str., Odessa, 65016, \\ Ukraine \\ E-mail: dirac13@mail.ru
}

